\title{
Trabeculectomy and optic nerve head topography
}

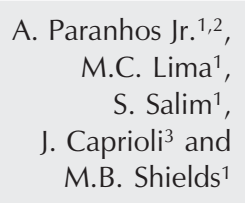

\author{
${ }^{1}$ Department of Ophthalmology and Visual Science, \\ Yale University, New Haven, CT, USA \\ ${ }^{2}$ Departamento de Oftalmologia, Universidade Federal de São Paulo, \\ São Paulo, SP, Brasil \\ ${ }^{3}$ Jules Stein Eye Institute, UCLA, Los Angeles, CA, USA
}

\begin{abstract}
Correspondence

A. Paranhos Jr.

Departamento de Oftalmologia

UNIFESP

Rua Botucatu, 824

04023-062 São Paulo, SP

Brasil

Fax: +55-11-5573-4002

E-mail: augusto.paranhos@uol.com.br

Research supported in part by an unrestricted grant from the

Connecticut Lions Eye Research

Foundation and Research to Prevent

Blindness.

Publication supported by FAPESP ......................

Received November 23, 2004 Accepted September 15, 2005

The objective of the present study was to evaluate changes in optic nerve head parameters, measured by confocal laser tomography, before and after trabeculectomy in order to identify outcome measures for the management of glaucoma. The optic nerve head of 22 eyes ( 22 patients) was analyzed by confocal laser tomography with the Heidelberg retinal tomogram (HRT) before and after trabeculectomy. The median time between the first HRT and surgery was 4.6 months (mean: $7.7 \pm 8.3$ ) and the median time between surgery and the second HRT was 10.8 months (mean: $12.0 \pm 6.8$ ). The patients were divided into two groups, i.e., those with the highest (group A) and lowest (group B) intraocular pressure (IOP) change after surgery. Differences in the 12 standard topographic parameters before and after surgery for each group were evaluated by the Wilcoxon signed rank test and the differences in these parameters between the two groups were compared by the Mann-Whitney rank sum test. Multiple regression analysis was used to evaluate the influence of the change in IOP $(\Delta \mathrm{IOP}$ and $\triangle \mathrm{IOP} \%$ ) and the changes in the other parameters. There were significant differences in the HRT measures before and after surgery in group A only for cup volume. In group B, no parameter was statistically different. The changes in group A were not significantly different than those in group B for any parameter $(\mathrm{P}>0.004$, Bonferroni correction for multiple comparisons). $\Delta \mathrm{IOP}$ and $\Delta \mathrm{IOP} \%$ had a statistically significant effect on $\Delta$ cup disk area, $\Delta$ cup volume and $\Delta$ mean cup depth. Changes in cup shape size were influenced significantly only by $\triangle \mathrm{IOP}$. Some optic disc parameters measured by HRT presented a significant improvement after filtering surgery, depending on the amount of IOP reduction. Long-term studies are needed to determine the usefulness of these findings as outcome measures in the management of glaucoma.
\end{abstract}

\section{Introduction}

The traditional outcome measure for adequacy of intraocular pressure (IOP) reduction with any medical or surgical interven-
Key words

- Optic nerve head

- Confocal laser tomography

- Intraocular pressure

- Trabeculectomy

- Glaucoma tion for glaucoma is the documentation of the stability or progression of optic neuropathy and visual field loss with time. The obvious disadvantage of this strategy is that irreversible damage must occur before the 
inadequacy of the therapy is recognized.

Although most glaucomatous damage is irreversible, some optic nerve head and visual field improvement following IOP reduction have been reported. The improvement has been associated with the amount of pressure reduction (1-3). Spaeth and associates $(4,5)$ have suggested that improvement in the appearance of the optic disc and/or visual field is an important early indicator of the long-term adequacy of glaucoma management. With the advent of technologies to more precisely and quantitatively measure changes in optic disc topography, investigators are beginning to take a closer look at the influence of lowering the IOP on disc morphology $(6,7)$.

The purpose of the present study was to quantitatively analyze changes in optic nerve head topography, as measured by confocal laser tomography before and after trabeculectomy, and by attempting to correlate the changes with the degree of IOP reduction.

\section{Material and Methods}

This is a retrospective study of patients who had undergone confocal laser tomography with the Heidelberg retinal tomogram (HRT, Software IR1-V2.01, Heidelberg Engineering, Heidelberg, Germany) before and after trabeculectomy. Only one eye of each patient was included in the study. If both eyes of one patient were eligible, data from the eye with the highest IOP reduction after surgery were used for analysis. Patients were excluded if HRT studies were thought to be unreliable or if they had other optic nerve disease in addition to glaucoma.

IOP in $\mathrm{mmHg}$ was measured with a Goldmann tonometer, and the absolute IOP change $(\triangle \mathrm{IOP})$ and the percent IOP reduction $(\triangle \mathrm{IOP} \%)$ before and after surgery were based on measurements obtained at the time of the HRT studies.

The principles and technique of confocal laser tomography with the HRT have been previously described $(8,9)$. The disc margin was defined by the contour line of the optic nerve head, which was drawn by the technician. For each image, the scanning depth was chosen by the operator, based on the depth of the optic disc cup. The mean of at least three images was used for the analyses of each exam.

A reference plane was defined as 0.05 $\mathrm{mm}$ posterior to the mean retinal surface at the disc margin near the papillo-macular bundle. Structures posterior to the plane were assigned to the optic nerve head cup, whereas structures anterior to the reference plan were regarded as the neuroretinal rim. The HRT stereometric parameters used in the analyses are listed in Table 1.

The patients were divided into two equal groups according to the amount of $\triangle \mathrm{IOP}$ or $\Delta \mathrm{IOP} \%$ using the median as the cut-off point. Both divisions gave coincident subgroups hence group $\mathrm{A}$ had higher $\triangle \mathrm{IOP}$ and $\triangle \mathrm{IOP} \%$ while group $\mathrm{B}$ had lower $\triangle \mathrm{IOP}$ and $\Delta \mathrm{IOP} \%$. Patient age and cup/disc area ratio before surgery and $\triangle \mathrm{IOP}, \Delta \mathrm{IOP} \%$ and the changes in HRT parameters after surgery were compared between the two groups using the Mann-Whitney rank sum test. The changes in HRT parameters before and after surgery for each group were evaluated by the Wilcoxon signed rank test. Non-parametric tests were used because of the high variability of all parameters in both groups.

Multiple regression analysis was also used with a backward stepwise regression strategy. In this analysis, the dependent variables were the differences of all stereometric parameters before and after surgery. The independent variables were $\Delta \mathrm{IOP}, \Delta \mathrm{IOP} \%$, the patient's age at the time of the surgery, and the cup/disc area ratio before surgery and the time between surgery and the last HRT exam. The variance inflation factor was used to evaluate colinearity of independent variables. Homoscedasticity and normality tests were also performed.

The study was performed at the Depart- 
ment of Ophthalmology, Yale University, New Haven, CT, USA, with the approval of the Yale Hospital Ethics Committee.

\section{Results}

Twenty-two eyes of 22 patients were included in this study. There were $15(68.2 \%)$ women. The ages ranged from 33 to 73 years (mean: $60.3 \pm 11.0$ ). Fourteen patients $(63.3 \%)$ had chronic open-angle glaucoma, $3(16.6 \%)$ had pigmentary glaucoma, 3 (16.6\%) had the pseudoexfoliation syndrome, and $2(9.1 \%)$ had low tension glaucoma. The median cup/disc area ratio before surgery, as measured by the HRT, was 0.52 (mean: 0.52 $\pm 0.21)$. The median time between the first HRT and surgery was 4.65 months (mean: $7.74 \pm 8.30)$ and the median time between surgery and the second HRT was 10.8 months (mean: $12.0 \pm 6.85$ ). In group A, the eleven patients had a median $\triangle \mathrm{IOP}$ of $11 \mathrm{mmHg}$ (mean: $13.45 \pm 6.14$ ) and a median $\triangle \mathrm{IOP} \%$ of $52.4 \%$ (mean: $52.21 \pm 15.46$ ), while the corresponding values for group B were 3 $\mathrm{mmHg}(2.36 \pm 2.69)$ and $26.7 \%(18.50 \pm$ 16.66), respectively. These differences between the two groups were statistically significant $(\mathrm{P}<0.0001)$. There were no significant differences between the two groups regarding the time of the HRT exam before or after surgery $(P=0.249$ and 0.247 , re- spectively), or the age or cup/disc area ratio ( $\mathrm{P}=0.9215$ and 0.0878 , respectively).

The measures of each HRT parameter before and after surgery and the comparisons between the changes in the two groups are listed in Table 1. There were significant differences between the HRT measures made before and after surgery in group A only for cup volume. In group B, none of the parameters was statistically different. The changes in group A were not significantly greater than those in group $\mathrm{B}$ for any parameter $(\mathrm{P}>$ 0.004, Bonferroni correction for multiple comparisons).

The stepwise regression analysis excluded age and cup/disc area ratio before surgery and the time between surgery and the last HRT exam as independent variables due to lack of a significant correlation with any change in HRT $(\mathrm{P}>0.05)$. Because $\Delta \mathrm{IOP} \%$ and $\Delta \mathrm{IOP}$ have a high colinearity, as indicated by the variance inflation factor (value of 4), the two variables were considered in different simple linear regression models. The results demonstrated that $\Delta \mathrm{IOP}$ and $\Delta \mathrm{IOP} \%$ have a statistically significant influence on $\Delta$ cup disc area, $\Delta$ cup volume and $\Delta$ mean cup depth. The change in cup shape measurements was significantly influenced only by $\triangle \mathrm{IOP}$ (Figure 1A-G). Linear regression analysis was considered to be appropriate by the normality and homoscedasticity tests $(\mathrm{P}>0.05)$.

Table 1. Heidelberg retinal tomogram parameters included in the analysis.

- Disk area $\left(\mathrm{mm}^{2}\right)$ : total area within the contour line

- Cup area $\left(\mathrm{mm}^{2}\right)$ : area below the reference plane

- Cup/disk area ratio: relation of the cup area and disk area (cup area/disk area)

- Rim area $\left(\mathrm{mm}^{2}\right)$ : area above the reference plane

- Height variation contour $(\mathrm{mm})$ : height variation of the retinal surface along the contour line (the height difference between the most elevated and the most depressed points of the contour line)

- Cup volume $\left(\mathrm{mm}^{3}\right)$ : volume below the reference plane

- Rim volume $\left(\mathrm{mm}^{3}\right)$ : volume above the reference plane

- Mean cup depth $(\mathrm{mm})$ : mean depth inside the contour line (relative to the curved surface)

- Maximum cup depth $(\mathrm{mm})$ : maximum depth inside the contour line

- Cup shape measure: measure of the overall three-dimensional shape of the cup

- Mean retinal nerve fiber layer (RNFL) thickness $(\mathrm{mm})$ : mean distance between the retinal surface along the contour line and the reference plane

- RNFL cross-sectional area $(\mathrm{mm})$ : mean distance between the retinal surface along the contour line and the reference plane, multiplied by the length of the contour line 

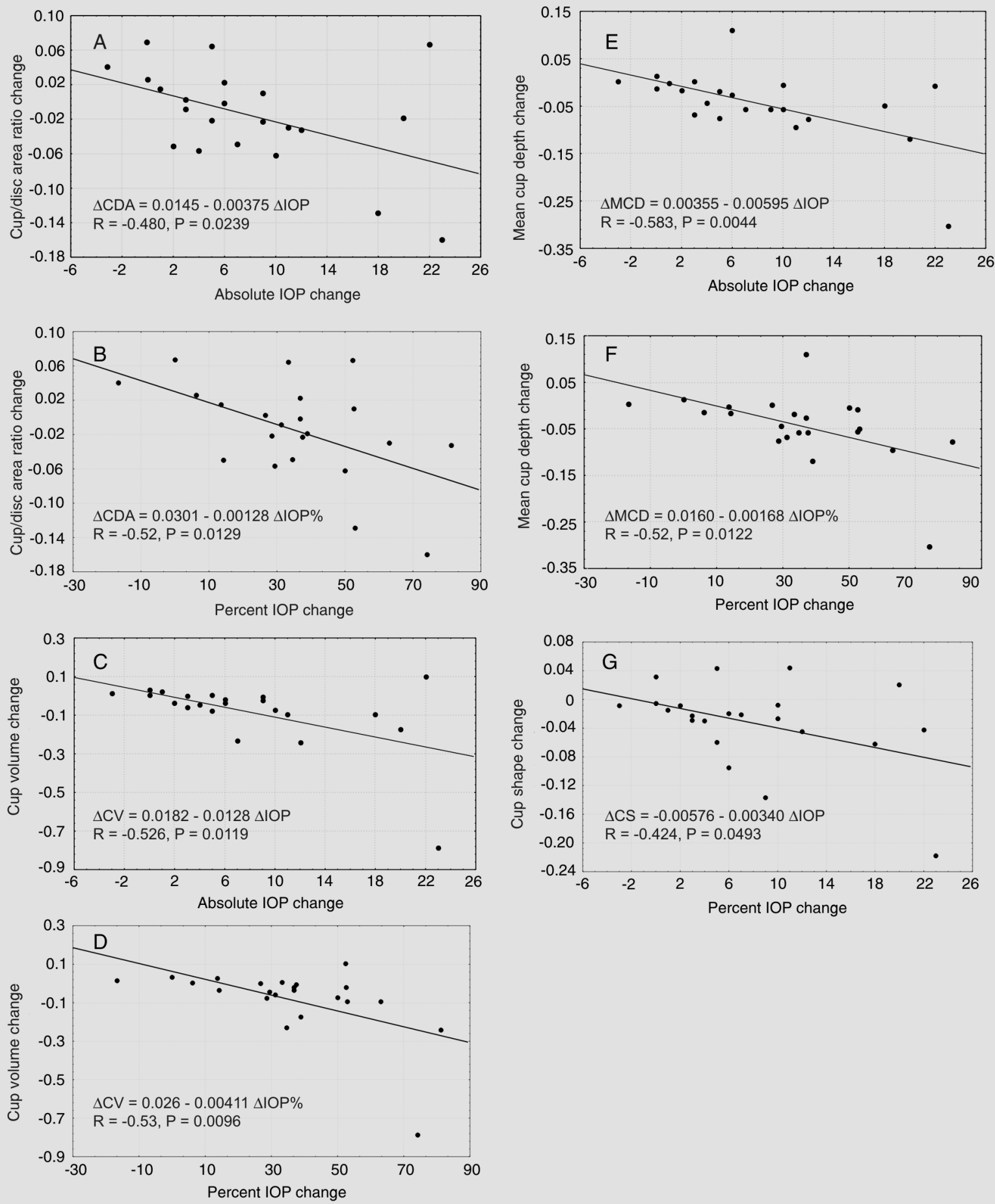

Figure 1. Effect of the reduction of intraocular pressure on optic nerve parameters after trabeculectomy. $A$, cup/disc area (CDA) ratio change and absolute interocular pressure (IOP) reduction; $B, \mathrm{CDA}$ ratio change and percent IOP reduction; $C$, cup volume (CV, mm ${ }^{3}$ ) change and absolute IOP reduction; $D$, cup volume $\left(\mathrm{mm}^{3}\right)$ and percent IOP reduction; $E$, mean cup depth (MCD) change (mm) and IOP reduction; $F$, mean cup depth change $(\mathrm{mm})$ and percent IOP reduction; $G$, cup shape (CS) change and absolute IOP reduction. Correlation coefficients were calculated by the method of Person. 


\section{Discussion}

An improvement in the appearance of the optic nerve head after reduction of IOP has been documented in children with glaucoma (10) and also in adults $(1,2,11)$. Improvement of the optic disc has been reported in adults after both surgical and medical reduction of IOP and in a variety of glaucomas with different techniques used to measure the changes (12).

Experimental studies suggest that apparent improvement in optic disc morphology may be due to compliance of the lamina cribrosa in response to changes in IOP. Levy and Crapps (13) demonstrated movement of the lamina in response to IOP change in an experimental model using human cadaver eyes in which platinum wires were placed in the lamina to allow radiographic observation of the displacement. Coleman et al. (14) also observed displacement of the optic nerve head in monkeys' eyes with a change in IOP and noted that this was more prominent in ocular hypertensive than normotensive animals, although there was considerable variability within each group. Shirakashi et al. (15) reported that the extent of cupping reversal after IOP reduction in primates with experimental glaucoma may decrease with progressive glaucomatous damage. Burgoyne et al. (12) also demonstrated reduced compliance of the optic disc in monkeys with chronic experimental glaucoma.

The variable compliance of the optic nerve head to changes in IOP may relate to ultrastructural differences in the lamina cribrosa. Hernandes (16) showed that elastin in the human lamina has ultrastructural differences in normal eyes as compared to those with glaucoma, and that this difference is influenced by age in normal eyes and by whether the glaucoma is early or advanced. In the early stages of chronic open-angle glaucoma, there appears to be new formation of elastin, while in the later stages, an abnormal enzymatic activity appears to en- hance degradation or impaired maturation of the newly formed material. In chronic openangle glaucoma, Hernandes (16) also found a decrease in collagen content, proliferation of basement membranes, and a change in elastic fibers with loss of the tubular structure.

Because of this apparent difference in the characteristics of the lamina cribrosa with age and the stage of glaucoma, we performed multiple regression analysis with the age of the patient and the cup/disc area ratio before surgery, using the latter as a parameter of the glaucoma severity. We did not find a significant correlation with either variable, although this could be due to an insufficient sample size to adequately represent different ages and levels of nerve damage. Shin et al. (17) and Topouzis et al. (18) also failed to find a significant association between reversal of cupping and age.

Another possible explanation for changes in the optic disc following IOP reduction with filtering surgery is edema of the nerve head and peripapillary retina, which has been described by Kawasaki and Purvin (19). Since this most likely occurs in the early postoperative period, it probably does not explain the disc changes in the present study, since the postoperative image analyses were performed an average of one year after surgery.

Whatever the mechanism of change in the optic disc following IOP reduction may be, it could offer a different approach to the management of glaucoma. Spaeth et al. $(4,5)$ noted that these changes obviously do not indicate that the disc has returned to normal in most cases, nor that they occur in every patient. However, the authors suggested that, if the ophthalmologist does not find improvement in the optic disc on careful examination after lowering the IOP, further lowering of the pressure should be considered.

One problem with this approach is that the traditional exam with stereo photography of the optic nerve head is not suffi- 
ciently accurate to detect a subtle improvement in all cases. The newer technologies of image analysis may improve our ability to detect these subtle changes. Shin et al. (17) reported that in 13 eyes with IOP reduction greater than $5 \mathrm{mmHg}, 10$ (77\%) had improvement in at least one optic disc parameter when analyzed by computerized stereophotogrammetry with the Rodenstock nerve head analyzer (Munich, Germany), while only 5 eyes $(38 \%)$ showed qualitative evidence of optic disc cup reversal with stereo photography. Lesk et al. (20) also found that the HRT was more sensitive in detecting changes in disc cupping than was the case with stereo photography.

The HRT, which is based on the principle of confocal laser tomography, appears to be a good tool to detect subtle improvement in the optic nerve since it has been shown to have good reproducibility $(8,9)$, has been used to demonstrate progression and improvement of the optic disc cupping with changes in IOP (21), and may be able to discriminate between persons with normal optic nerve heads and those with early glaucomatous damage (22).

Using HRT technology in the present study, we found statistical improvement after IOP reduction in optic disc parameters related only to cup volume in group A, which had relatively greater IOP reduction, and none in group B, which had less IOP reduction. Other topographic parameters improved in group A, but did not reach statistical significance, probably because of lack of power to detect these differences. The sample size is to small to find significant differences for most of the HRT parameters and larger studies or meta-analyses are needed to clarify the real influence of the IOP on HRT parameters. Nevertheless, the findings of some outliers with big differences in HRT parameters after surgery should be a concern in clinical practice.

We have confirmed and extended the observations of other investigators. Irak et al. (6) used the HRT to evaluate changes in the optic nerve head in 49 patients after trabeculectomy and found improvement in the same parameters as in the present study, as well as in rim area, rim volume and height variation contour. With the exception of rim area, these latter parameters also improved in our study, although the change was not statistically significant. Other investigators have also reported improvement in the optic disc parameters, as measured with the HRT, after lowering the IOP $(7,20,23)$.

Topouzis et al. (18) suggested that the improvement in disc morphology following IOP reduction is time-limited, since the changes at 2 weeks after surgery did not persist 4 and 8 months later, with the exception of cup shape, which was still improved by the fourth month. However, Shin et al. (17) reported that the time after IOP reduction did not correlate with changes in the optic disc, and Raitta et al. (23) found statistically significant improvement in cup volume and mean cup depth after a mean follow-up of 3.7 months, which remained significant for cup volume at 12.1 months. In the latter study, cup/disc area ratio and mean height contour, which were not significantly changed at the first follow-up, were significantly improved in the later exam. In our study, the longer follow-up between surgery and the second HRT of $12.0 \pm 6.85$ months suggests some degree of stability in the parameters that improved after IOP reduction, as also does the nonsignificant correlation with the improvement of any parameter and the time of follow-up. However, the lack of shorter-term follow-up is a limitation of the present study, and further studies are needed to clarify the stability of improvement in optic disc parameters following a sustained reduction of IOP.

In the present study, regression analysis showed that $\triangle \mathrm{IOP}$ and $\triangle \mathrm{IOP} \%$ can statistically influence changes in disc parameters as measured by the HRT. This correlation has also been reported by others $(17,20)$, 
although some have found a less consistent correlation $(18,24)$.

The most important result presented here is that, although the correlation with the $\triangle \mathrm{IOP}$ and $\triangle \mathrm{IOP} \%$ and optic disc changes is statistically significant, it probably has no influence on clinical evaluation of the disc and the damage progression as most of all parameters did not change significantly.

The important question that remains to be answered is whether improvement in one or more disc parameters in association with a particular degree of IOP reduction is a reliable indicator that the new pressure level, assuming it remains constant, will be adequate to prevent further glaucomatous optic neuropathy. The same question must also be applied to visual function tests. Large population, long-term, prospective trials with both functional and morphologic tests after IOP reduction are needed to determine whether these outcome measures can improve our ability to manage glaucoma.

\section{References}

1. Pederson JE \& Herschler J (1982). Reversal of glaucomatous cupping in adults. Archives of Ophthalmology, 100: 426-431.

2. Greenidge KC, Spaeth GL \& Traverso CE (1985). Change in appearance of the optic disc associated with lowering of intraocular pressure. Ophthalmology, 92: 897-903.

3. Tsai CS, Shin DH, Wan JY et al. (1991). Visual field global indices in patients with reversal of glaucomatous cupping after intraocular pressure reduction. Ophthalmology, 98: 1412-1419.

4. Spaeth GL, Fellman RL, Starita RL et al. (1985). A new management system for glaucoma based on improvement of the appearance of the optic disc or visual field. Transactions of the American Ophthalmological Society, 83: 268-284.

5. Spaeth GL (1988). A new management system for glaucoma based on improvement of the appearance of the optic disc or visual field. Fortschritte der Ophthalmologie, 85: 614-619.

6. Irak I, Zangwill L, Garden V et al. (1996). Change in optic disk topography after trabeculectomy. American Journal of Ophthalmology, 122: 690-695.

7. Park KH, Kim DM \& Youn DH (1997). Short-term change of optic nerve head topography after trabeculectomy in adult glaucoma patients as measured by Heidelberg retina tomograph. Korean Journal of Ophthalmology, 11: 1-6.

8. Weinreb RN, Lusky M, Bartsch DU et al. (1993). Effect of repetitive imaging on topographic measurements of the optic nerve head. Archives of Ophthalmology, 111: 636-638.

9. Rohrschneider K, Burk ROW, Kruse FE et al. (1994). Reproducibility of the optic nerve head topography with a new laser topographic scanning device. Ophthalmology, 101: 1044-1049.

10. Quigley HA (1982). Childhood glaucoma. Results with trabeculotomy and study of reversible cupping. Ophthalmology, 89: 219-226.

11. Spaeth GL (1989). Reversibility of optic disc cupping. Archives of Ophthalmology, 107: 1583-1584.

12. Burgoyne CF, Quigley HA, Thompson HW et al. (1995). Early changes in optic disc compliance and surface position in experimental glaucoma. Ophthalmology, 102: 1800-1809.
13. Levy N \& Crapps EE (1984). Displacement of optic nerve head in response to short-term intraocular pressure elevation in human eyes. Archives of Ophthalmology, 102: 782-786.

14. Coleman AL, Quigley HA, Vitale S et al. (1991). Displacement of the optic nerve head by acute changes in intraocular pressure in monkey eyes. Ophthalmology, 98: 35-40.

15. Shirakashi M, Nanba K \& Iwata K (1992). Changes in reversal cupping in experimental glaucoma. Ophthalmology, 99: 1104-1110.

16. Hernandes MR (1992). Ultrastructural immunocytochemical analysis of elastin in the human lamina cribrosa. Investigative Ophthalmology and Visual Science, 33: 2891-2903.

17. Shin DH, Bielik M, Hong JY et al. (1989). Reversal of glaucomatous optic disc cupping in adult patients. Archives of Ophthalmology, 107: 1599-1603.

18. Topouzis F, Peng F, Kotas-Neumann R et al. (1999). Longitudinal changes in optic disc topography of adult patients after trabeculectomy. Ophthalmology, 106: 1147-1151.

19. Kawasaki A \& Purvin V (1998). Unilateral optic disc edema following trabeculectomy. Journal of Neuro-Ophthalmology, 18: 121-123.

20. Lesk MR, Spaeth GL, Azuara-Blanco A et al. (1999). Reversal of optic disc cupping after glaucoma surgery analyzed with a scanning laser tomograph. Ophthalmology, 106: 1013-1018.

21. Azura-Blanco A, Harris A, Cantor LB et al. (1998). Effects of short term increase of intraocular pressure on optic disc cupping. British Journal of Ophthalmology, 82: 880-883.

22. Uchida H, Brigatti L \& Caprioli J (1996). Detection of structural damage from glaucoma with confocal laser image analysis. Investigative Ophthalmology and Visual Science, 37: 2393-2401.

23. Raitta C, Tomita G, Vesti E et al. (1996). Optic disc topography before and after trabeculectomy in advanced glaucoma. Ophthalmic Surgery and Lasers, 27: 349-354.

24. Funk J \& Grehn F (1989). Changes in the temporal neuroretinal border area of the papilla following pressure-reducing surgery. Fortschritte der Ophthalmologie, 86: 727-731. 